\title{
Resolving Vibrational from Electronic Coherences in Two-Dimensional Electronic Spectroscopy: The Role of the Laser Spectrum
}

\author{
Franco V. de A. Camargo, ${ }^{1,2}$ Lena Grimmelsmann, ${ }^{1,3}$ Harry L. Anderson, ${ }^{4}$ Stephen R. Meech, ${ }^{1}$ and Ismael A. Heisler ${ }^{1}$ \\ ${ }^{1}$ School of Chemistry, Norwich Research Park, University of East Anglia, Norwich NR4 7TJ, United Kingdom \\ ${ }^{2}$ CAPES Foundation, Ministry of Education of Brazil, Brasilia DF 70040-202, Brazil \\ ${ }^{3}$ Fakultät für Chemie und Biochemie, Ruhr-Universität Bochum, \\ Universitätsstrasse 150, 44801, Bochum, Germany \\ ${ }^{4}$ Department of Chemistry, Chemistry Research Laboratory, \\ University of Oxford, Oxford OX1 3TA, United Kingdom
}

(Dated: December 5, 2016)

\begin{abstract}
The observation of coherent quantum effects in photosynthetic light-harvesting complexes prompted the question whether quantum coherence could be exploited to improve the efficiency in new energy materials. The detailed characterization of coherent effects relies on sensitive methods such as two-dimensional electronic spectroscopy (2D-ES). However, the interpretation of the results produced by $2 \mathrm{D}-\mathrm{ES}$ is challenging due to the many possible couplings present in complex molecular structures. In this work, we demonstrate how the laser spectral profile can induce electronic coherence-like signals in monomeric chromophores, potentially leading to data misinterpretation. We argue that the laser spectrum acts as a filter for certain coherence pathways and thus propose a general method to differentiate vibrational from electronic coherences.
\end{abstract}

The development of two-dimensional electronic spectroscopy (2D-ES) enabled photophysical processes to be resolved with unprecedented temporal and spectral detail. The extra dimension, given by the excitation frequency axis, yields 2D spectra where discrimination of specific features (otherwise overlapped in 1D methods) is now possible [1]. Consequently 2D-ES has provided new insights into topics as diverse as quantum phenomena in biology [2-4], energy transfer [5, 6], singlet fission [7], nanomaterials $[8,9]$, reaction dynamics [10-12], and many body effects in coupled quantum wells [13-15]. However, although 2D-ES greatly facilitates the discrimination of physical phenomena affecting electronic transitions such as line-broadening mechanisms, vibrational and electronic couplings, ambiguities remain in detailed analysis, especially for molecular systems where both vibrational and electronic couplings may play a role.

The observation of long-lived oscillations in 2D-ES measurements, assigned to coherent coupling between electronic transitions, in light harvesting systems sparked major interest, as it raised questions such as whether coherent effects could underlie the remarkably high efficiency of energy transfer in photosynthesis [16]. However, electronic transitions are commonly coupled to molecular vibrational modes, and vibrational coherences both in the ground and excited states can be prepared by broadband pulses, in the same way that a coherent superposition of different excitonic or electronic states may be prepared [17]. Recent theoretical and experimental research has explored the different coherence signatures arising in 2D$\mathrm{ES}$, but for the most part possible effects from the laser spectrum have been neglected [18-24].

In this Letter we study vibrational coherences in a zincporphyrin monomer (where electronic couplings are absent), and find that the laser spectrum content plays a crucial role in what is observed. We show that a laser spectrum with characteristics typical of those generally used in 2D-ES generates results which mimic the coherent evolution expected from a purely excitonic coupling scheme. This result is of critical importance in designing and interpreting 2D-ES experiments on coupled systems. We describe the physical origin of these results, and how such spectral filtering effects can be used in practice as a general method to unambiguously characterize vibrational coherences separately from other phenomena.

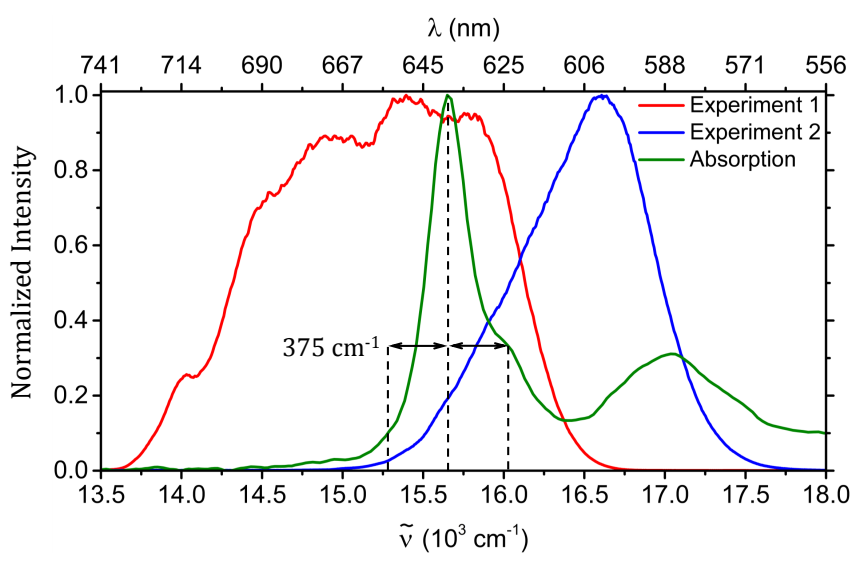

Figure 1. Normalized linear absorption (green) and laser spectra used in experiments 1 (red) and 2 (blue). The sample was dissolved in $n$-pentane with $1 \%$ by volume of pyridine to a concentration of $\sim 330 \mu \mathrm{M}$ in a $200 \mu \mathrm{m}$ pathlength static cell, corresponding to an optical density of 0.25 at $15650 \mathrm{~cm}^{-1}$ $(639 \mathrm{~nm})$.

In order to study purely vibrational coherences in 2DES we chose a 5,15-bisalkynyl zinc porphyrin monomer [25], the normalized linear absorption of which is shown in Figure 1. The lowest electronic transition in por- 
phyrins is to a singlet state labelled the $Q$ band and here it contains 2 peaks. The peak at $15650 \mathrm{~cm}^{-1}$ corresponds to the $Q_{x}(0-0)$ band [26], which is coupled to a zinc-pyrrole vibrational breathing mode of the porphyrin ring at $375 \mathrm{~cm}^{-1}$, resulting in a vibronic shoulder at $16025 \mathrm{~cm}^{-1}$ [27]. The second absorption peak at higher energies $\left(\tilde{\nu}>16500 \mathrm{~cm}^{-1}\right)$ includes the $Q_{y}(0-0)$ transition and higher energy vibrational modes coupled to $Q_{x}$ [28]. Thus the electronic structure for $\tilde{\nu}<16500 \mathrm{~cm}^{-1}$ can be adequately described by a displaced harmonic oscillator model representing one electronic transition coupled to a single vibrational mode, as shown in Figure $2 \mathrm{a}$.

We performed 2D-ES experiments using a conventional optics based scheme, described in the SM, and detailed in reference [29]. 2D-ES requires determination of the excitation axis, which is possible by experimentally controlling the time delay between the first and the second field-matter interactions (labeled $\tau$ - the coherence time), the Fourier pair of which is the excitation wavenumber $\tilde{\nu}_{1}$. Further, 2D-ES recovers the full complex-valued third-order polarization $P^{(3)}\left(\tilde{\nu}_{1}, T, \tilde{\nu}_{3}\right)$, which is the convolution of the system's third-order response function $S^{(3)}\left(\tilde{\nu}_{1}, T, \tilde{\nu}_{3}\right)$ with the electric fields [30], where the resolution of $\tilde{\nu}_{1}$ is defined only by the $\tau$ scanning range, and the temporal resolution along $T$ (the population time) remains defined by the pulse duration [31]. As a result, 2DES recovers correlation maps between excitation and detection $\left(\tilde{\nu}_{1}, \tilde{\nu}_{3}\right)$ for each $T$. The $2 \mathrm{D}$-ES signal arises from four-wave mixing pathways, which can be either rephasing (photon echo signal) or non-rephasing (free induction decay signal) according to their phase-matching, and an equally weighed sum of both results in $P^{(3)}\left(\tilde{\nu}_{1}, T, \tilde{\nu}_{3}\right)$ $[32,33]$. Our experiment recovers complex-valued rephasing and non-rephasing maps independently [29, 34].

In this work we are specifically concerned with fourwave mixing pathways which include vibrational coherences either in the ground or electronic excited states during $T$. For the displaced harmonic oscillator model, considering only the energy levels explicitly drawn in Figure 2a there are 16 such pathways (Figures S3-S7 display all [26]) and they generate oscillatory features in the data as a function of $T$. It is known that the phase of the oscillation due to each single pathway is a function of $\left(\tilde{\nu}_{1}, \tilde{\nu}_{3}\right)$, and that interference between neighboring contributions can generate confounding features, so isolation of as few pathways as possible is helpful for proper interpretation of the data $[18,34]$. To that end, we note that 8 of the coherence pathways are rephasing, while the other 8 are non-rephasing. Further, we note that the oscillatory behavior arises from the systems' density matrix being in a coherence (such as $\left|g_{0}\right\rangle\left\langle g_{1}\right|$ or $\left|g_{1}\right\rangle\left\langle g_{0}\right|$ ) during $T$. The density matrix is a Hermitian operator, so $\left|g_{0}\right\rangle\left\langle g_{1}|=| g_{1}\right\rangle\left\langle\left. g_{0}\right|^{*}\right.$, and therefore, if $\left.\mid g_{0}\right\rangle\left\langle g_{1}\right|$ modulates the third order response as $e^{+i \nu_{0} T}$, then $\left|g_{1}\right\rangle\left\langle g_{0}\right|$ will modulate the response as $e^{-i \nu_{0} T}$, where $\nu_{0}>0$. Conse-

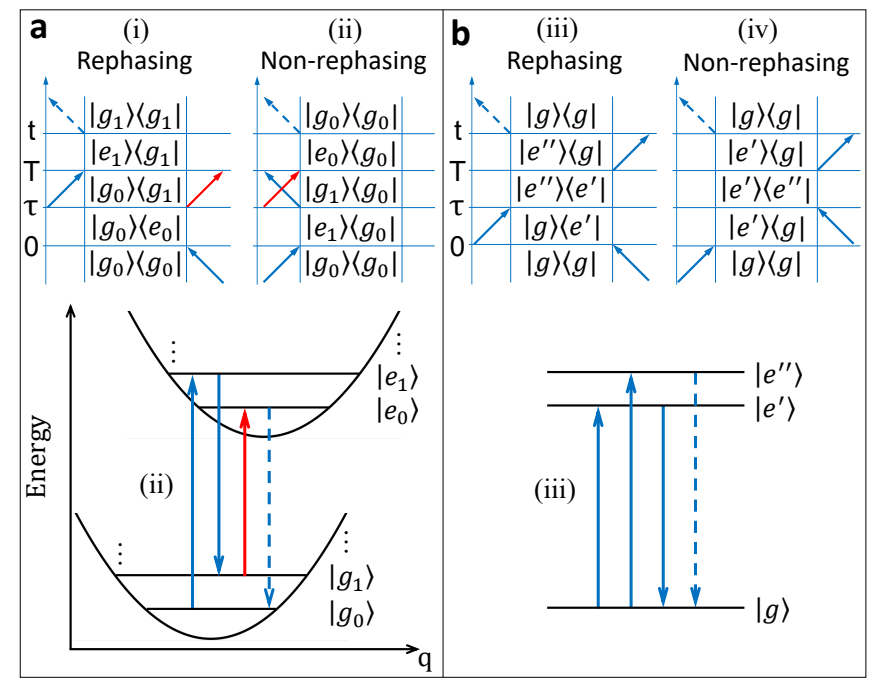

Figure 2. (a) Examples of rephasing (i) and non-rephasing (ii) pathways with ground state coherences during $T$. The first arrow defines $\tilde{\nu}_{1}$, the dashed arrow is signal emission $\left(\tilde{\nu}_{3}\right)$ and arrows connecting $\left|g_{1}\right\rangle$ to $\left|e_{0}\right\rangle$ are red. Below, pathway (ii) is drawn in a displaced harmonic oscillator energy level scheme. (b) Similar to (a), for an energy level with two electronic excited states. Here, pathways similar to (i) and (ii) are not possible, but in (a) pathways like (iii) and (iv) are.

quently, beatings arising from $\left|g_{0}\right\rangle\left\langle g_{1}\right|$ and $\left|g_{1}\right\rangle\left\langle g_{0}\right|$ coherences during $T$ will be observed with opposite frequency signs when the complex-valued spectra are Fourier transformed [35]. Thus we can sort the pathways for each of rephasing and non-rephasing signals according to $\tilde{\nu}_{1}$ (first arrow), $\tilde{\nu}_{3}$ (dashed arrow) and the frequency sign during $T$, obtaining Figures 3a,b. Significantly, rephasing negative (Figure 3a, red symbols) and non-rephasing positive (Figure 3b, green symbols) frequencies include only two pathways, each forming 2 peaks, all corresponding to vibrational coherences in the electronic excited state [36]. The remaining 6 pathways for both rephasing and nonrephasing have frequencies with opposite sign and are arranged in 4 peaks (Figures 3a,b).

The first set of measurements was performed using the laser spectrum displayed in red in Figure 1. This spectrum fully covers the lowest peak in the sample's linear absorption and also has significant bandwidth towards lower energies. Our 2D-ES setup allows fast acquisition of complex-valued rephasing and non-rephasing spectra, enabling us to scan $T$ from 30 to 1000 fs with time steps of 5 fs, yielding 196 spectra of each type. These maps include not only the the oscillatory pathways discussed above, but also an equal number of non-oscillatory ones, which are associated with population dynamics [26]. In order to remove non-oscillatory amplitude, we stack together 2D-ES maps for all values of $T$ and globally fit the data with exponential decays, so that the residuals from this fit will consist only of the coherent oscillations [37]. We then perform a Fourier Transform of the complex- 


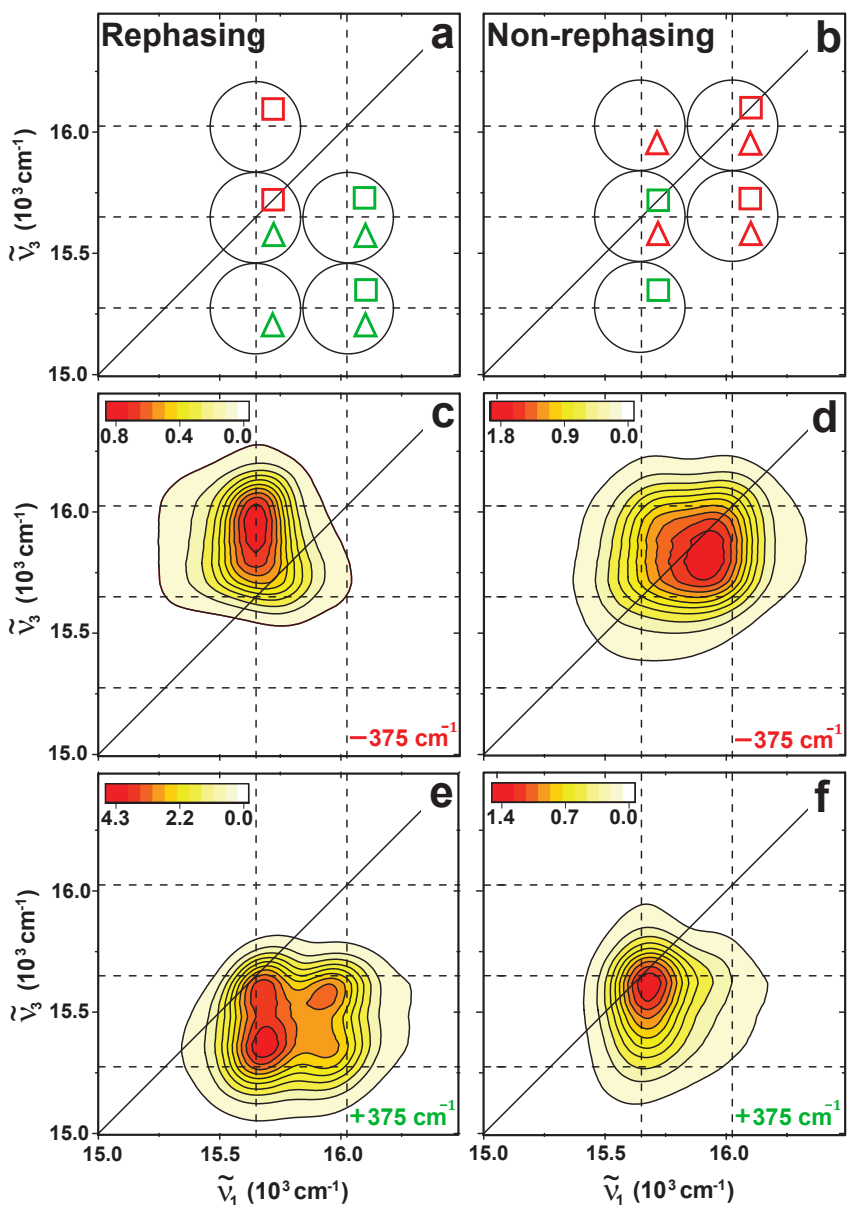

Figure 3. (a) Diagrammatic scheme representing the positions of the rephasing oscillatory pathways in the $\left(\tilde{\nu}_{1}, \tilde{\nu}_{3}\right)$ plane. Each oscillatory pathway is marked with an open geometric figure, with triangles and squares representing vibrational coherences in the electronic ground and excited states respectively. Red and green symbols represent pathways of negative and positive frequency respectively, and the 2DES signal from each pathway is centered on the black circle around them. (b) Same as (a) for non-rephasing. (cf) Experimentally obtained oscillation amplitude maps for $\tilde{\nu}_{2}=-375 \mathrm{~cm}^{-1}$ in rephasing (c) and non-rephasing (d) measurements, and $\tilde{\nu}_{2}=+375 \mathrm{~cm}^{-1}$ in rephasing (e) and nonrephasing (f) in experiment 1.

valued residuals of rephasing and non-rephasing signals over $T$ for every $\left(\tilde{\nu}_{1}, \tilde{\nu}_{3}\right)$ pair, thus recovering the oscillatory amplitude over $T$ for any given wavenumber $\tilde{\nu}_{2}$ within the Nyquist limit. By selecting a specific value of $\tilde{\nu}_{2}$, we can plot the corresponding amplitude as a function of $\left(\tilde{\nu}_{1}, \tilde{\nu}_{3}\right)$, which are called oscillation amplitude maps [38]. Because they include only the oscillatory amplitude at the selected value of $\tilde{\nu}_{2}$, they are analogous to the schemes in Figures 3a,b. The experimental oscillation amplitude maps at $\tilde{\nu}_{2}= \pm 375 \mathrm{~cm}^{-1}$ are shown in Figures 3c-f. We observe good agreement between experimental results and the the predictions in Figures 3a,b. For example, Figure 3e has four peaks forming a square approximately in the positions marked by the green symbols in Figure 3a, while Figure 3c shows amplitude elongated along the positions marked by the red symbols in Figure 3a. Such agreement confirms that the experiment is capable of resolving positive from negative frequencies (see Figure S15 for typical time domain data [26]). Even though we maximized the isolation of pathways, overlap between neighboring contributions in the $\left(\tilde{\nu}_{1}, \tilde{\nu}_{3}\right)$ plane remains present, leading to the convoluted nature of the peaks and small shifts from the predicted $\left(\tilde{\nu}_{1}, \tilde{\nu}_{3}\right)$ coordinates [34].

These oscillation maps contrast with those expected for an electronically coupled system with energy levels shown in Figure 2b, in which case the absence of a second energy level in the ground state significantly reduces the number of pathways. For instance, coherence pathways analogous to (iii) and (iv) in Figure $2 \mathrm{~b}$ are possible in the vibrational case, but none like (i) and (ii) can exist in the pure electronic coupling case, as they require a sub-level in the ground state [18]. In the electronic case only four coherence pathways remain, with two oscillatory peaks in the diagonal in non-rephasing maps, and two in the off-diagonal in rephasing maps [26]. Thus, the collective presence of pathways in either this pattern or that from Figures 3c-f is a good criterion to distinguish purely vibrational from purely electronic coherences [39].

At this point, we emphasize that there is a significant difference between the present experiment and the usual cases in 2D-ES. Here, the molecule's absorption peak is sufficiently narrow to be fully covered by the laser spectrum with substantial bandwidth remaining on the low energy side. Most systems of interest with absorption in the visible region possess much broader absorption peaks, which are challenging to cover even with the shortest available pulses. Thus a typical 2D-ES experiment centers the laser spectrum around the absorption region of interest, leaving little bandwidth to lower wavenumbers than the pure electronic transition, where there is no absorption. Since the early days in ultrafast spectroscopy authors have been aware of potential effects due to the laser spectrum [40], and seminal 2D-ES studies concluded it merely acts as a filter on the $\tilde{\nu}_{1}$ and $\tilde{\nu}_{3}$ axes [41, 42] which was experimentally confirmed [21]. However, these studies focused on 2D-ES maps for a single $T$, not discussing coherent signatures. Therefore we performed a second set of 2D-ES measurements using the spectrum shown in blue in Figure 1, once again selecting only the oscillatory features at $375 \mathrm{~cm}^{-1}$ using the same procedure as for Figure 3.

To discuss the results expected for experiment 2, we note that although the spectrum fully covers the lowest absorption peak, it has almost no amplitude at $15275 \mathrm{~cm}^{-1}$, corresponding to a transition between $\left|e_{0}\right\rangle$ and $\left|g_{1}\right\rangle$ (see Figures 1 and 2a). It follows that the oscillatory features with detection at $15275 \mathrm{~cm}^{-1}$ will be suppressed, as the detection axis $\left(\tilde{\nu}_{3}\right)$ range is defined by 

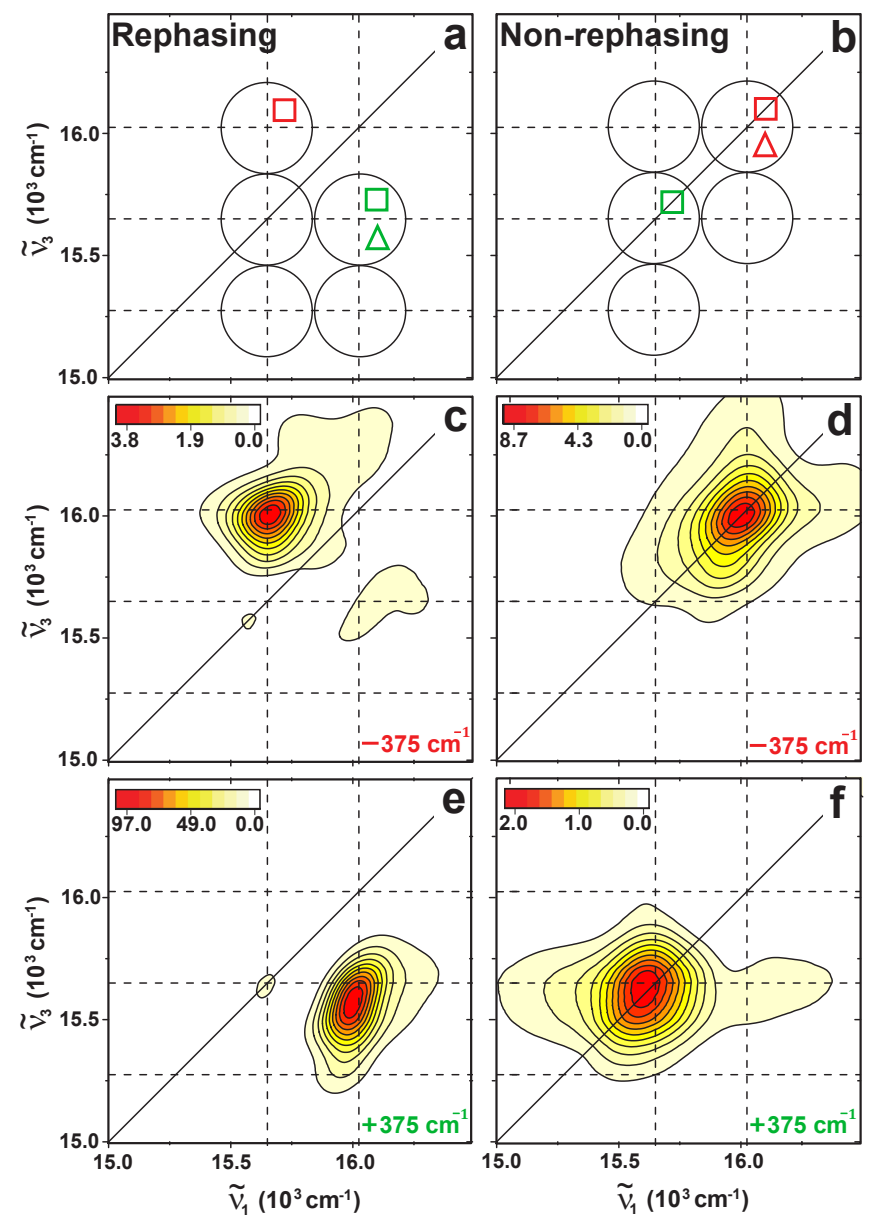

Figure 4. Same as Figure 3 for experiment 2.

the laser spectrum. Regarding the excitation axis $\left(\tilde{\nu}_{1}\right)$, it is defined by the first field-matter interaction, which can only happen at a frequency which the molecule absorbs. Therefore, because all absorption is still covered by the laser, there is no effect on this axis. From these considerations, it is tempting to merely exclude the peaks detected at $15275 \mathrm{~cm}^{-1}$ from Figure 3 and consider the rest will remain unaltered. However, 2D-ES signals arise from three field-matter interactions before signal emission, and all interactions must be at frequencies covered by the laser spectrum. This requires the exclusion of all pathways for which any of the field-matter interactions take place at a frequency not present in the laser spectrum. For example, pathways (i) and (ii) shown in Figure 2a are both suppressed because an intermediate field-matter interaction (red arrows) takes place at $15275 \mathrm{~cm}^{-1}$, even though excitation and detection frequencies lie within the laser spectrum. This plays an important role regarding which vibrational coherences are detected in 2D-ES experiments. The coherence pathways with all interactions wholly within the laser spectrum of experiment 2 are represented in Figures 4a,b. The surprising result is not only that the remaining oscillatory peaks are di- agonal for non-rephasing and off-diagonal for rephasing, but also that the frequency sign of the oscillation is the same as that expected for a purely electronic coherence (see Figure S11 [26]). The experimental result in Figures $4 \mathrm{c}-\mathrm{f}$ confirms this forecast. Therefore, in a typical realization of a $2 \mathrm{D}$ study in the visible region, a vibrational mode coupled to an electronic transition may generate the same pattern of oscillatory features as an electronic coherence, potentially misleading interpretation.

The key issue is that a spectrum like that of experiment 1, covering a vibrational quantum on both sides of the main absorption, is often unattainable in practice, so only a spectrum similar to experiment 2 is used. Having understood the role of the laser spectrum allows us to go one step further and consider a new experiment, using a red-shifted laser spectrum which covers the transitions between $\left|g_{0}\right\rangle$ and $\left|e_{0}\right\rangle$, and between $\left|e_{0}\right\rangle$ and $\left|g_{1}\right\rangle$, but fails to cover the transition between $\left|g_{0}\right\rangle$ and $\left|e_{1}\right\rangle$. This means that the vibronic peak in the linear absorption is not covered, and therefore no coherences in the electronic excited state can be prepared (as an excitation to $\left|e_{1}\right\rangle$ would be required). Nonetheless, systematic analysis shows that two coherence pathways in the ground state remain: one non-rephasing, analogous to that represented in Figure $3 \mathrm{~b}$ as a red triangle at $\tilde{\nu}_{1}=\tilde{\nu}_{3}=15650 \mathrm{~cm}^{-1}$; and one rephasing, analogous to the green triangle at $\tilde{\nu}_{1}=15650 \mathrm{~cm}^{-1}$ and $\tilde{\nu}_{3}=15275 \mathrm{~cm}^{-1}$ in Figure 3a (see Figure S9 [26]).

To test this, we note that the porphyrin monomer is known to have a vibrational mode at $1340 \mathrm{~cm}^{-1}$ coupled to $Q_{x}$, for which experiment 1 falls exactly in this regime: the vibronic peak in the linear absorption is at $16990 \mathrm{~cm}^{-1}$ [28], where there is no laser intensity, but intensity is available at $\tilde{\nu}_{3}=15650-1340 \mathrm{~cm}^{-1}=$ $14310 \mathrm{~cm}^{-1}$. Figure 5 shows the rephasing oscillation amplitude map at $\tilde{\nu}_{2}=+1340 \mathrm{~cm}^{-1}$ with the scale zoomed around $\tilde{\nu}_{3}=14310 \mathrm{~cm}^{-1}$, and a peak is indeed detected. Figures $5 \mathrm{~b}$ and $\mathrm{c}$ show the time and frequency domain data at this coordinate, confirming the expected oscillation. We emphasize that the rephasing amplitude of this oscillation is smaller than $0.4 \%$ of the maximum signal, but can still be resolved because it is a cross peak in a mostly background free region. In the non-rephasing diagonal, where the other pathway is expected, the signal to noise ratio of our measurement was insufficient to detect this oscillation.

In conclusion, the present results deepen the understanding on how to detect and interpret coherent oscillations in 2D-ES measurements, highlighting the fact that for complex energy level structures, the laser spectrum plays a crucial and non trivial role regarding the oscillations detected. We have demonstrated that a redshifted laser spectrum can be used to selectively prepare coherences in the ground state, thus complementing experiments where the spectrum causes the ambiguity we reported. The effect of the laser spectrum on coher- 

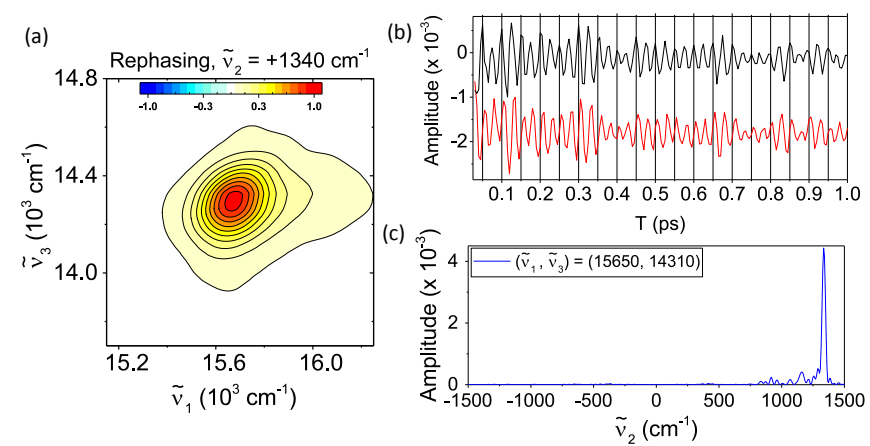

Figure 5. (a) Oscillation amplitude map at $\tilde{\nu}_{2}=1340 \mathrm{~cm}^{-1}$. (b) Real (black) and imaginary (red) rephasing amplitude at $\tilde{\nu}_{1}=15650 \mathrm{~cm}^{-1}$ and $\tilde{\nu}_{3}=14310 \mathrm{~cm}^{-1}$ in experiment 1 . (c) Power spectrum of the fit residuals at the same position. The amplitude at negative frequencies is negligible and the smaller peaks arise from Raman modes of $n$-pentane [43].

ences has been recently discussed in the literature, but the filtering effect was not discussed [44]. The selection of specific pathways through two-color experiments has also been described [45-47], but the concept that some vibronic signatures may be missing in one-color experiments, as described here, was previously only briefly noted by Butkus et al. [48]. Recently, there have been efforts to model excitonic systems with the inclusion of vibrational coupling, giving rise to more complex energy level structures [49], which were experimentally verified in an artificial light harvesting complex whose structure proved especially convenient for rigorous polarization experiments [50]. For the study of other complex systems, our results demonstrate that explicit inclusion of the laser spectrum is essential for rigorous design and interpretation of 2D-ES experiments, and show how the laser spectrum can be used as a tool to fully benchmark groundstate coherences.

This work was supported by EPSRC grants EP/J009148/1 and J021431/1. Franco V. A. Camargo thanks Brazilian Funding Agency CAPES for his doctoral studentship (BEX 9527/13-3). Lena Grimmelsmann thanks the Cluster of Excellence RESOLV (EXC 1069) funded by the Deutsche Forschungsgemeinschaft for the financial support. We thank Professor Darius Abramavicius and Dr. Vytautas Butkus for useful discussions, and Dr. Martin D. Peeks for synthesizing the porphyrin monomer used in this work.

[1] J. D. Hybl, A. W. Albrecht, S. M. Gallagher Faeder, and D. M. Jonas, Chemical Physics Letters 297, 307 (1998).

[2] H. Lee, Y.-C. Cheng, and G. R. Fleming, Science 316, 1462 (2007).

[3] G. S. Engel, T. R. Calhoun, E. L. Read, T. K. Ahn, T. Mancal, Y. C. Cheng, R. E. Blankenship, and G. R.
Fleming, Nature 446, 782 (2007).

[4] E. Collini, C. Y. Wong, K. E. Wilk, P. M. G. Curmi, P. Brumer, and G. D. Scholes, Nature 463, 644 (2010).

[5] E. Thyrhaug, K. Žídek, J. Dostál, D. Bína, and D. Zigmantas, The Journal of Physical Chemistry Letters 7, 1653 (2016).

[6] E. Collini and G. D. Scholes, Science 323, 369 (2009).

[7] A. A. Bakulin, S. E. Morgan, T. B. Kehoe, M. W. B. Wilson, A. W. Chin, D. Zigmantas, D. Egorova, and A. Rao, Nature Chemistry 8, 16 (2016).

[8] E. Cassette, J. C. Dean, and G. D. Scholes, Small 12, 2234 (2016).

[9] T. Stoll, E. Sgrò, J. W. Jarrett, J. Réhault, A. Oriana, L. Sala, F. Branchi, G. Cerullo, and K. L. Knappenberger, Journal of the American Chemical Society 138, 1788 (2016).

[10] P. Nuernberger, S. Ruetzel, and T. Brixner, Angewandte Chemie - International Edition 54, 11368 (2015).

[11] S. Ruetzel, M. Kullmann, J. Buback, P. Nuernberger, and T. Brixner, Physical Review Letters 110, 148305 (2013).

[12] F. V. A. Camargo, H. L. Anderson, S. R. Meech, and I. A. Heisler, Journal of Physical Chemistry B 119, 14660 (2015).

[13] K. W. Stone, K. Gundogdu, D. B. Turner, X. Li, S. T. Cundiff, and K. A. Nelson, Science 324, 1169 (2009).

[14] G. Nardin, G. Moody, R. Singh, T. M. Autry, H. Li, F. Morier-Genoud, and S. T. Cundiff, Physical Review Letters 112, 046402 (2014).

[15] G. Moody, I. A. Akimov, H. Li, R. Singh, D. R. Yakovlev, G. Karczewski, M. Wiater, T. Wojtowicz, M. Bayer, and S. T. Cundiff, Physical Review Letters 112, 097401 (2014).

[16] A. Chenu and G. D. Scholes, Annual Review of Physical Chemistry 66, 69 (2015).

[17] N. Christensson, F. Milota, J. Hauer, J. Sperling, O. Bixner, A. Nemeth, and H. F. Kauffmann, Journal of Physical Chemistry B 115, 5383 (2011).

[18] V. Butkus, D. Zigmantas, L. Valkunas, and D. Abramavicius, Chemical Physics Letters 545, 40 (2012).

[19] V. Butkus, D. Zigmantas, D. Abramavicius, and L. Valkunas, Chemical Physics Letters 587, 93 (2013).

[20] T. Mancal, N. Christensson, V. Lukes, F. Milota, O. Bixner, H. F. Kauffmann, and J. Hauer, Journal of Physical Chemistry Letters 3, 1497 (2012).

[21] J. R. Caram, A. F. Fidler, and G. S. Engel, The Journal of Chemical Physics 137, 024507 (2012).

[22] A. Chenu, N. Christensson, H. F. Kauffmann, and T. Mancal, Scientific Reports 3, 2029 (2013).

[23] V. Perlík, C. Lincoln, F. Šanda, and J. Hauer, The Journal of Physical Chemistry Letters 5, 404 (2014).

[24] E. Cassette, R. D. Pensack, B. Mahler, and G. D. Scholes, Nature Communications 6, 6086 (2015).

[25] D. Koszelewski, A. Nowak-Krol, M. Drobizhev, C. J. Wilson, J. E. Haley, T. M. Cooper, J. Romiszewski, E. Gorecka, H. L. Anderson, A. Rebane, and D. T. Gryko, Journal of Materials Chemistry C 1, 2044 (2013).

[26] See Supplemental Material at [URL will be inserted by publisher] for sample details, experimental description, four wave mixing pathways, examples of real and imaginary rephasing and non-rephasing maps, and typical time domain data for experiments 1 and 2 .

[27] M. Atamian, R. J. Donohoe, J. S. Lindsey, and D. F. Bocian, The Journal of Physical Chemistry 93, 2236 (1989). 
[28] M. Drobizhev, Y. Stepanenko, Y. Dzenis, A. Karotki, A. Rebane, P. N. Taylor, and H. L. Anderson, The Journal of Physical Chemistry B 109, 7223 (2005).

[29] I. A. Heisler, R. Moca, F. V. A. Camargo, and S. R. Meech, Review of Scientific Instruments 85, 063103 (2014).

[30] A. Tokmakoff, The Journal of Physical Chemistry A 104, 4247 (2000).

[31] P. Hamm and M. Zanni, Concepts and methods of $2 D$ infrared spectroscopy (Cambridge University Press, 2011).

[32] M. Khalil, N. Demirdöven, and A. Tokmakoff, Physical Review Letters 90, 047401 (2003).

[33] M. Khalil, N. Demirdöven, and A. Tokmakoff, Journal of Physical Chemistry A 107, 5258 (2003).

[34] F. V. A. Camargo, H. L. Anderson, S. R. Meech, and I. A. Heisler, The Journal of Physical Chemistry A 119, 95 (2015).

[35] J. Seibt and T. Pullerits, The Journal of Physical Chemistry C 117, 18728 (2013).

[36] Y. Song, C. Hellmann, N. Stingelin, and G. D. Scholes, The Journal of Chemical Physics 142, 212410 (2015).

[37] V. I. Prokhorenko, EPA Newsletter , 21 (2012).

[38] D. B. Turner, R. Dinshaw, K.-K. Lee, M. S. Belsley, K. E. Wilk, P. M. G. Curmi, and G. D. Scholes, Physical Chemistry Chemical Physics 14, 4857 (2012).

[39] Y.-C. Cheng and G. R. Fleming, Journal of Physical Chemistry A 112, 4254 (2008).

[40] A. Lorincz, F. A. Novak, and S. A. Rice, Chemical
Physics Letters 111, 322 (1984).

[41] S. M. G. Faeder and D. M. Jonas, Journal of Physical Chemistry A 103, 10489 (1999).

[42] P. Kjellberg, B. Bruggemann, and T. Pullerits, Physical Review B $\mathbf{7 4}$ (2006).

[43] C. D. Keefe and S. Jaspers-fayer, Vibrational Spectroscopy $\mathbf{5 7}, 72$ (2011).

[44] R. Tempelaar, A. Halpin, P. J. M. Johnson, J. Cai, R. S. Murphy, J. Knoester, R. J. D. Miller, and T. L. C. Jansen, The Journal of Physical Chemistry A , 3042 (2016).

[45] D. Abramavicius, V. Butkus, J. Bujokas, and L. Valkunas, Chemical Physics 372, 22 (2010).

[46] S. S. Senlik, V. R. Policht, and J. P. Ogilvie, Journal of Physical Chemistry Letters 6, 2413 (2015).

[47] J. O. Tollerud, C. R. Hall, and J. A. Davis, Optics Express 22, 6719 (2014).

[48] V. Butkus, A. Gelzinis, R. Augulis, A. Gall, C. Büchel, B. Robert, D. Zigmantas, L. Valkunas, and D. Abramavicius, The Journal of Chemical Physics 142, 212414 (2015).

[49] V. Tiwari, W. K. Peters, and D. M. Jonas, Proceedings of the National Academy of Sciences 110, 1203 (2013).

[50] J. Lim, D. Paleček, F. Caycedo-Soler, C. N. Lincoln, J. Prior, H. von Berlepsch, S. F. Huelga, M. B. Plenio, D. Zigmantas, and J. Hauer, Nature Communications 6, 7755 (2015). 